\title{
HD 191110 a SB2 system with HgMn and $\mathrm{Hg}$ components Orbital elements and abundance analysis
}

\author{
G. Catanzaro ${ }^{1}$, F. Leone ${ }^{1, \star}$, and P. Leto ${ }^{2}$ \\ 1 INAF - Catania Astrophysical Observatory, Via S. Sofia 78, 95123 Catania, Italy \\ e-mail: gca@ct.astro.it; fleone@ct.astro.it \\ 2 Istituto di Radioastronomia del C.N.R., Stazione VLBI di Noto, CP 161 Noto, Italy \\ e-mail: pleto@noto.ira.cnr.it
}

Received 14 April 2003 / Accepted 22 May 2003

\begin{abstract}
In this paper we present the first quantitative abundance analysis of the SB2 system with $\mathrm{HgMn}$ and $\mathrm{Hg}$ components HD 191110. Time-resolved $(R=14000)$ spectroscopic observations of this system have been obtained during September and October 2002 at the Catania Astrophysical Observatory. Combining these observations with spectra $(R=50000-110000)$ taken from the $C F H T$ archive, we refined the orbital period of this system $(P=9.34661 \pm 0.00002 \mathrm{~d})$ and then we recalculated the fundamental parameters characterizing its orbit.

From the fit of the $\mathrm{H}_{\beta}$ profiles, obtained at different orbital phases, we determined the effective temperatures and surface gravities of each component and the light ratio: $T_{\text {eff }}^{A}=11000 \mathrm{~K}, \log g^{A}=3.60, T_{\text {eff }}^{B}=10700 \mathrm{~K}, \log g^{B}=3.90, L_{\mathrm{A}} / L_{\mathrm{B}}=1.20$.

Regarding the chemical composition, both components have solar abundances of sulfur, titanium and iron; magnesium and silicon are slightly underabundant; platinum and yttrium are overabundant. Manganese lines have been detected only in the spectrum of the primary. As to rare-earths, two lines of NdIII have been identified in the secondary's spectrum. Other lines we identified in our spectra belong to PII, CrII, NiII, SrII, ZrII and AuII. We also discuss the isotopic structure of the HgII $\lambda 3983.9 \AA$ line.
\end{abstract}

Key words. stars individual: HD 191110 - binaries: spectroscopic - stars: abundances - stars: early-type -

stars: chemically peculiar

\section{Introduction}

HgMn stars in binary systems play an important role in understanding the formation of the chemical peculiarities. As many of these systems have components sufficiently far apart to have evolved independently from their protostellar cloud, they could be used to study how the peculiarities originate in their atmospheres and how they evolve.

HD 191110 (= HR 7694 = AV Cap) is a double-lined spectroscopic binary with $\mathrm{HgMn}$ and $\mathrm{Hg}$ components; the first mention of its binary nature was given by Adams \& Joy (1917). It was classified as B9.5III by Cowley et al. (1969). Later, Dworetsky (1974) computed the first orbital solution for the system. He did not undertake a detailed abundance analysis, but noted the presence of a strong $\operatorname{HgII} \lambda 3984$ line in both

Send offprint requests to: G. Catanzaro, e-mail: gca@ct . astro. it

* Guest User, Canadian Astronomy Data Centre, which is operated by the Dominion Astrophysical Observatory for the National Research Council of Canada's Herzberg Institute of Astrophysics. components, MnII lines in the primary's spectrum only and YII and PtII $\lambda 4046$ lines only in the secondary's spectrum.

Despite the importance and rarity of SB2 systems where both components are $\mathrm{Hg}$ stars, no quantitative abundance analysis on HD 191110 have been performed since the preliminary work by Dworetsky (1974).

Within our program dedicated to the determination of the orbital parameters of binaries in which one or both components are Chemically Peculiar (CP) stars, we have obtained timeresolved spectroscopic observations of HD 191110. Combining CFHT archive data of this system with our spectra, we also performed a detailed abundance analysis of the two components.

\section{Observations and data reduction}

Radial velocities and chemical abundances for HD 191110 have been derived from spectra obtained with two different instruments:

- the $0.9 \mathrm{~m}$ telescope of the Catania Astrophysical Observatory (OAC), which is fiber-linked to a REOSC 
echelle spectrograph, has been used to obtain time-resolved spectroscopy of HD 191110 in the 4810-5370 ̊ spectral region. We observed this system for 3 consecutive nights on September 17th, 18th and 19th 2002 and for 2 nights on October 18th and 19th 2002. The resolving power deducted from the lines of the Th-Ar lamp is $\approx 14000$.

- the $3.6 \mathrm{~m}$ telescope of the CFHT equipped with the $f / 4$ coudé (Gecko) spectrograph. A summary of the instrumental setup for the four nights is given in Table 1, and includes the dates of the observations, the observed spectral regions and the resolutions as deduced from the FWHM of Th-Ar comparison lamp lines.

The stellar spectra, calibrated in wavelength and with the continuum normalized to a unity level, were obtained using standard data reduction procedures for spectroscopic observations within the NOAO/IRAF package. The achieved S/N was always above 300 for the CFHT spectra and $\approx 100$ for the OAC data.

Equivalent widths have been measured with a gaussian fit of each line using standard IRAF routines. As the main source of errors in the equivalent width measurements is the uncertain position of the continuum we applied the formula given in Leone et al. (1995), which takes into account the width of the line and the rms of the continuum. According to $\mathrm{S} / \mathrm{N}$ of our spectra and to the rotational velocities estimated for HD 191110 A \& B, we found that the typical uncertainty is $\approx 1 \mathrm{mÅ}$ or even less.

To calculate radial velocities we first identified all unblended spectral lines using Kurucz's (1993) line list. For each of these lines we measured the central wavelength with a gaussian fit of the profile and we computed the radial velocity using the classical Doppler shift formula. Finally we averaged the values obtained for each night. Mean radial velocity and the corresponding $\sigma$ have been reported in Table 2. For the night Sept. 10th, 1995 (HJD = 2449970.902) this procedure could not be used since the stars were close to conjunction. Thus, radial velocity has been measured by modeling the observed spectra according to the procedures described in Sects. 4 and 5 .

Table 1. Journal of CFHT observations.

\begin{tabular}{ccr}
\hline \hline Date & $\begin{array}{c}\text { Sp. range } \\
\lambda(\AA)\end{array}$ & \multicolumn{1}{c}{$R$} \\
& \multicolumn{1}{c}{$\lambda / \Delta \lambda$} \\
\hline Sept. 07, 1995 & $3770-3795$ & 60000 \\
& $4040-4065$ & 110000 \\
Sept. 08, 1995 & $3760-3800$ & 60000 \\
& $3970-3995$ & 95000 \\
& $4040-4065$ & 110000 \\
Sept. 09, 1995 & $4150-4180$ & 50000 \\
& $4490-4530$ & 90000 \\
& $4900-4940$ & 55000 \\
Sept. 10, 1995 & $3750-3780$ & 50000 \\
& $4020-4045$ & 110000 \\
\hline
\end{tabular}

To evaluate the systematic errors in the radial velocities measured at OAC, during our observing runs we observed stars with constant and well known radial velocity: HD 693, HD 3712 and HD 223368 extracted from the list of CORAVEL standard stars (Udry et al. 1999). For each of these stars we computed the average radial velocity and standard deviation. Systematic errors were derived from the average difference between radial velocity values from CORAVEL and our measurements as equal to $2.50 \pm 1.37 \mathrm{~km} \mathrm{~s}^{-1}$. The measured radial velocities of our program star listed in Table 2 have been corrected for systematic errors.

Following the nomenclature first used by Dworetsky (1974), we defined the primary as the star showing the Mn lines.

\section{The determination of orbital parameters}

The radial velocities for a spectroscopic binary system are given from the following equation:

$V_{\mathrm{rad}}=\gamma+K[\cos (\theta+\omega)+e \cos \omega]$

where $\gamma$ is the radial velocity of the center of mass, $e$ is the eccentricity of the orbit, $\omega$ is the longitude of the periastron, $\theta$ is the angular position of the star measured from the center of mass at a given instant and $K$ is the semi-amplitude of the velocity curve as given by the formula:

$K=\frac{2 \pi a \sin i}{P \sqrt{1-e^{2}}}$

where $P$ is the orbital period of the system.

Radial velocity measurements of HD 191110 have been reported by Dworetsky (1974) and by Aikman (1976). Combining these velocities with those derived from our spectra, orbital elements have been determined by a weighted leastsquares fitting to Eq. (1). Errors have been estimated as the variation in the parameters which increases the $\chi^{2}$ of a unit. The starting value for $P$ was evaluated using the Phase Dispersion

Table 2. Heliocentric Julian Date and measured radial velocities for HD 191110, $n$ represents the number of spectral lines used.

\begin{tabular}{cccccc}
\hline \hline & HJD & \multicolumn{5}{c}{$R V\left(\mathrm{~km} \mathrm{~s}^{-1}\right)$} \\
& $(2400000.0+)$ & Primary & $n$ & Secondary & $n$ \\
\hline CFHT & 49967.897 & $36.94 \pm 0.67$ & 8 & $-60.63 \pm 0.63$ & 8 \\
& 49968.810 & $43.12 \pm 0.65$ & 18 & $-66.12 \pm 0.91$ & 15 \\
& 49969.948 & $23.78 \pm 0.97$ & 27 & $-44.70 \pm 0.61$ & 13 \\
& 49970.902 & $-6.74 \pm 0.75$ & & $-10.21 \pm 0.75$ & \\
\hline OAC & 52535.330 & $-45.45 \pm 1.88$ & 9 & $36.84 \pm 3.42$ & 9 \\
& 52536.355 & $-14.22 \pm 1.26$ & 9 & $-4.05 \pm 1.26$ & 9 \\
& 52537.361 & $19.09 \pm 3.98$ & 4 & $-31.31 \pm 4.56$ & 4 \\
& 52566.232 & $38.16 \pm 0.55$ & 10 & $-56.88 \pm 1.26$ & 10 \\
& 52567.288 & $45.64 \pm 1.81$ & 9 & $-60.33 \pm 1.23$ & 10 \\
\hline
\end{tabular}




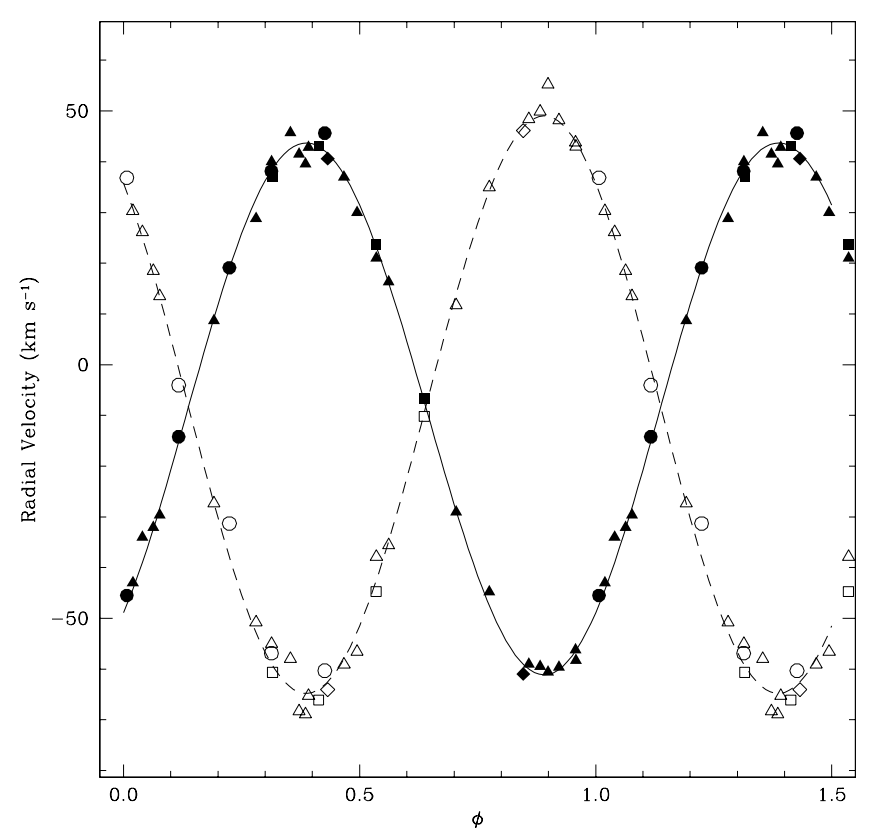

Fig. 1. Radial velocity curves for the primary (solid line and filled symbols) and the secondary (dashed line and open symbols). Our measurements are represented by circles (OAC) and squares (CFHT), Dworetsky (1974) data by triangles and Aikman (1976) data by rhombs.

Method (Stellingwerf 1978) as coded in the NOAO/IRAF package. We obtained the following solution:

$\begin{array}{ll}P: & 9.34661 \pm 0.00002 \mathrm{~d} \\ T: & 2452572.651 \pm 0.023 \\ e: & 0.01 \pm 0.02 \\ \omega: & 220.0^{\circ} \pm 1.0^{\circ} \\ K_{\mathrm{A}}: & 52.42 \pm 0.99 \mathrm{~km} \mathrm{~s}^{-1} \\ K_{\mathrm{B}}: & 56.91 \pm 1.10 \mathrm{~km} \mathrm{~s}^{-1} \\ \gamma: & -8.31 \pm 0.56 \mathrm{~km} \mathrm{~s}^{-1} \\ a \sin i_{\mathrm{A}}: & 9.68 \pm 0.18 R_{\odot} \\ a \sin i_{\mathrm{B}}: & 10.51 \pm 0.20 R_{\odot} \\ m_{\mathrm{A}} / m_{\mathrm{B}}: & 1.08 \pm 0.04 .\end{array}$

Since we obtained a very small eccentricity from our fitting procedure, we tested if this small value is significant or not. Lucy \& Sweeney (1971) developed a criterion for deciding when to reject an elliptical orbit in favor of a circular one.

As for HD 191110, we conclude that an elliptical orbit is significant only at the 5\% confidence level and therefore a circular orbit should be assigned to HD 191110.

After having fixed $e=0$ we repeated the fitting procedure obtaining orbital parameters always coincident, within the experimental errors, with those previously reported.

Our solution gives a slightly longer period than the one published by Dworetsky (1974). However, our mass ratio is in very good agreement with his value. Figure 1 shows the radial velocity curves for both components of HD 191110 where different data sets are marked with different symbols.

Giménez \& Zamorano (1985) gave a useful equation to estimate the ratio of the radii from a given mass ratio:

$\log R_{\mathrm{A}} / R_{\mathrm{B}}=0.556 \cdot \log m_{\mathrm{A}} / m_{\mathrm{B}}$.
Table 3. Derived values of effective temperatures, gravities and light ratio $\left(L_{\mathrm{A}} / L_{\mathrm{B}}\right)$ for HD $191110 \mathrm{~A} \& \mathrm{~B}$. We report the parameters achieved for a solar helium abundance and those computed for helium abundances equal to those actually observed.

\begin{tabular}{lcccc}
\hline \hline & \multicolumn{2}{c}{$\mathrm{He}=\odot$} & \multicolumn{2}{c}{$\mathrm{He}=*$} \\
& $\chi^{2}=1.12$ & \multicolumn{2}{c}{$\chi^{2}=1.14$} \\
& $T_{\text {eff }}$ & $\log g$ & $T_{\text {eff }}$ & $\log g$ \\
\hline Primary & 11800 & 3.70 & 11000 & 3.60 \\
Secondary & 11000 & 4.00 & 10700 & 3.90 \\
Light ratio & \multicolumn{2}{c}{1.19} & \multicolumn{2}{c}{1.20} \\
\hline
\end{tabular}

From the previous equation we derived a ratio of the radii equal to $1.04 \pm 0.04$.

\section{Stellar parameters}

One method commonly used to determine effective temperature $\left(T_{\text {eff }}\right)$ and surface gravity $(\log g)$ of a star is to compare the observed and theoretical profiles of a Balmer line. In the case of the SB2 system, we observe $\mathrm{H}_{\beta}$ profiles given by the superposition of lines Doppler-shifted by the orbital motion and weighted by the different luminosity.

The procedure used for HD 191110 was to minimize the difference among observed and synthetic $\mathrm{H}_{\beta}$ profiles obtained at five different orbital phases. This procedure took into account simultaneously the spectra of the two components and the light ratio between them. A grid of atmospheric models has been calculated with ATLAS9 (Kurucz 1993) and the synthetic $\mathrm{H}_{\beta}$ with SYNTHE (Kurucz \& Avrett 1981). The grid extends over the intervals $9000 \mathrm{~K} \leq T_{\text {eff }} \leq 15000 \mathrm{~K}$ (step of $100 \mathrm{~K}$ ) and $3.5 \leq \log g \leq 4.5$ (step $0.1 \mathrm{dex}$ ). For each pair of models we changed the light ratio $\left(L_{\mathrm{A}} / L_{\mathrm{B}}\right)$ in the range $0.5-2.0$ (step 0.01).

To reduce the number of parameters, rotational velocities of HD 191110 A \& B have been determinated by fitting CFHT highest resolution lines with synthetic profiles computed for $v \sin i$ from 2 to $10 \mathrm{~km} \mathrm{~s}^{-1}$ with a step of $0.5 \mathrm{~km} \mathrm{~s}^{-1}$. The best fit occurs for a rotational velocity of $7 \mathrm{~km} \mathrm{~s}^{-1}$ for both components.

As a result of $\mathrm{H}_{\beta}$ fitting we obtained the parameters reported in the first column of Table 3. These values of $T_{\text {eff }}, \log g$ and light ratio, obtained assuming solar abundances of helium and metals, have been used as starting values for an iterative procedure necessary to derive the stellar parameters consistent with the abundances. Leone \& Manfrè (1996) showed the importance of a correct helium and metal abundance assumption in determining the effective temperature and gravity of stars. Particularly, helium can be largely underabundant in $\mathrm{HgMn}$ stars as shown by several analyses reported in the literature (see for example Table 7 in Adelman 1998).

To fix the helium abundance in both components we considered the spectral region centered around HeI $\lambda 4921 \AA$ observed at the CFHT and reported in Fig. 3. A synthetic spectrum computed using SYNTHE showed that this line is practically at the noise level in both components. Then, in view of the $\mathrm{S} / \mathrm{N}$, a value of $\log \mathrm{He} / N_{\text {tot }}=-1.39$ for the primary and $\log \mathrm{He} / N_{\text {tot }}=-2.00$ for the secondary could be considered as upper limits for the helium abundances. 


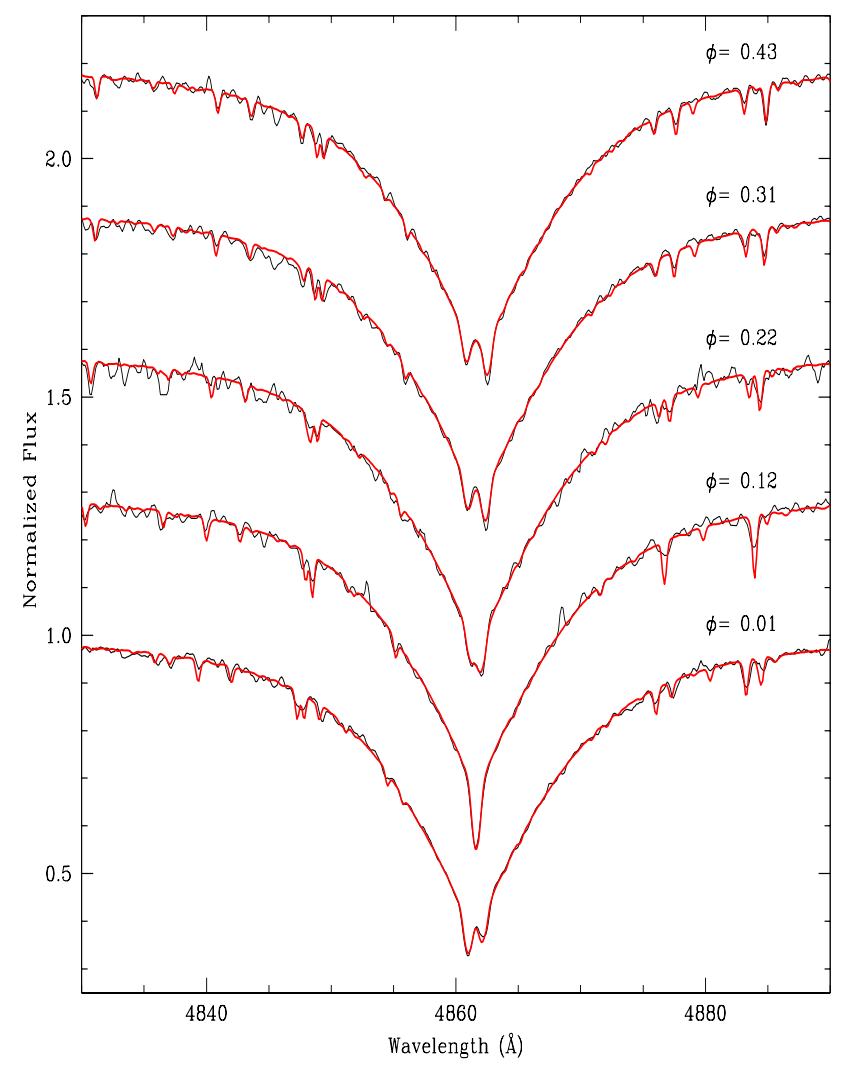

Fig. 2. Comparison between the observed and computed $\mathrm{H}_{\beta}$ line profiles. Above each profile we give the corresponding orbital phase.

Hence, we then repeated the iterative procedure, considering a new grid of models computed with the adopted helium underabundances. This new calculation give us the values reported in the last two columns of Table 3 . The previous helium abundances are confirmed also for the new atmosphere models. Errors in $T_{\text {eff }}$ and $\log g$ have been estimated as the variation in the parameters which increases the $\chi^{2}$ by one unit. We found $\delta T \approx 200 \mathrm{~K}$ and $\delta \log g \approx 0.1 \mathrm{dex}$, values consistent with the step adopted in our grid of models. As to metal opacity we adopted the solar one, being metal abundances not very different than in the Sun.

As a consistency check we calculated the luminosity ratio using the formula:

$\frac{L_{\mathrm{A}}}{L_{\mathrm{B}}}=\left(\frac{R_{\mathrm{A}}}{R_{\mathrm{B}}}\right)^{2} \cdot\left(\frac{T_{\mathrm{eff}, \mathrm{A}}}{T_{\mathrm{eff}, \mathrm{B}}}\right)^{4}$

where the ratio of the radii is that calculated from Eq. (3) and the temperatures are those derived from fitting $\mathrm{H}_{\beta}$ profiles. Replacing the corresponding values in Eq. (4) we obtained $1.21 \pm 0.20$, a value very close to the adopted luminosity ratio derived spectroscopically.

Figure 2 shows the observed and calculated $\mathrm{H}_{\beta}$.

\section{Abundance analysis}

We adopted the $T_{\text {eff }}, \log g$ and light ratio values given in Table 3 to derive the abundances of components. In general, the light ratio is a function of wavelength. In the case of HD 191110,

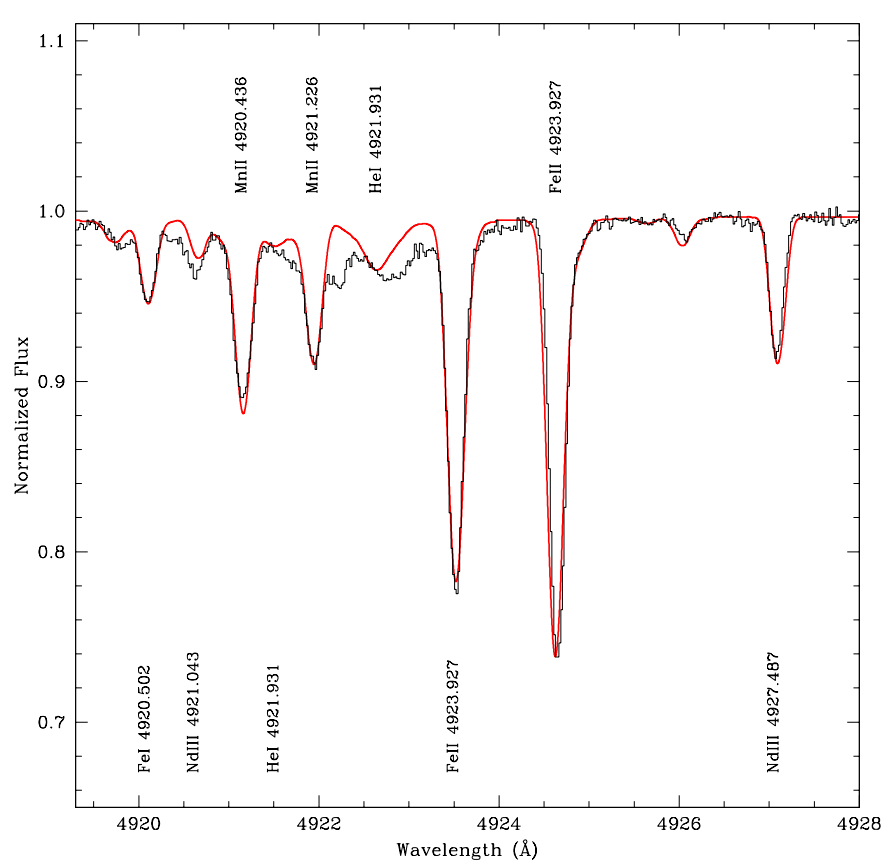

Fig. 3. Synthetic spectra (solid line) of the region centered around $\lambda 4923 \AA$ compared with the observations (histogram). As described in Sect. 4, the HeI $\lambda 4921$ lines are confused at the noise level for both components. The line MnII $\lambda 4920.436$ is actually blended with FeI $\lambda 4920.502$. Labels above the continuum refer to primary and those below the spectrum to the secondary.

the spectral types of the components are so similar that we may conclude that the wavelength dependence is negligible. However, in order to quantify the previous statement, we calculated the ratio between the synthetic fluxes computed with ATLAS9 for the adopted $T_{\text {eff }}$ and $\log g$. In the spectral range of our interest, the light ratio is constant to within $0.01 \%$.

In Table A.1 we report for each component of HD 191110: laboratory wavelengths of identified spectral lines, observed and corrected equivalent widths and derived abundances. For comparison we reported also the adopted standard abundances from Grevesse et al. (1996).

To correct the observed equivalent widths for the light ratio $l=L_{\mathrm{A}} / L_{\mathrm{B}}$, we used the equation (Conti 1970):

$W_{\mathrm{A}}^{\mathrm{corr}}=\frac{1+l}{l} \cdot W_{\mathrm{A}}^{\mathrm{obs}}$

for the primary component and the equation

$W_{\mathrm{B}}^{\mathrm{corr}}=(1+l) \cdot W_{\mathrm{B}}^{\mathrm{obs}}$

for the secondary component.

For line identification we used Kurucz's (1993) atomic line list for all elements with the exception of PtII, AuII and HgII. Atomic parameters for PtII and AuII are from the VALD database (Piskunov et al. 1995). At the end of this section, we will discuss in detail the isotopic structure of HgII observed in HD 191110.

As a first step, we determined the microturbulence of each star using 16 FeII unblended lines, by requiring that abundances derived line by line do not depend on equivalent widths. The 


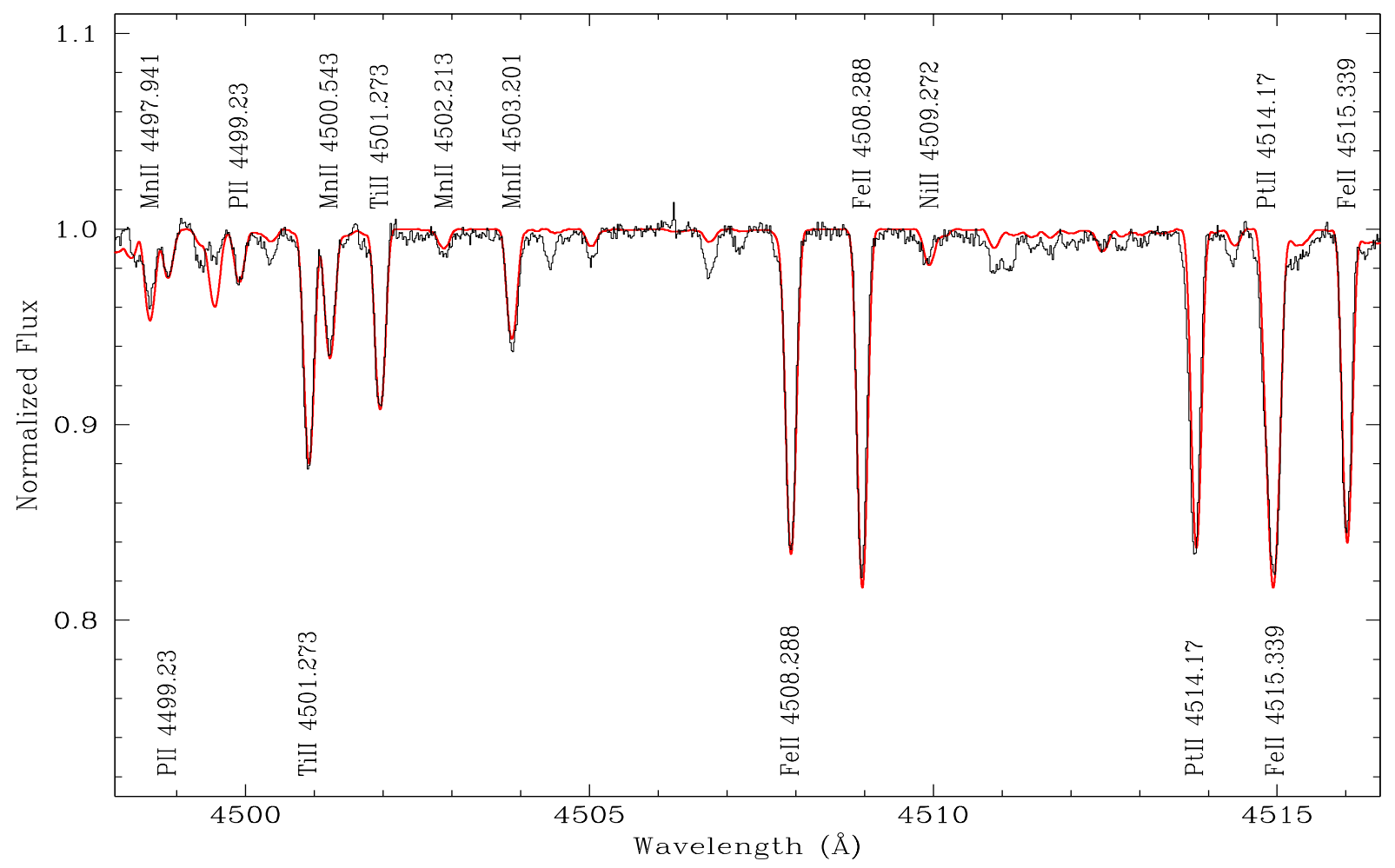

Fig. 4. Comparison of the observed spectrum in the region 4500-4517 $\AA$ with synthetic spectrum computed using the inferred metal abundances. In this plot labels above the continuum refer to primary and those below the spectrum to the secondary.

microturbulent velocities obtained for HD $191110 \mathrm{~A}$ and B are $1.7 \pm 0.3 \mathrm{~km} \mathrm{~s}^{-1}$ and $1.8 \pm 0.3 \mathrm{~km} \mathrm{~s}^{-1}$ respectively .

The abundance analysis has been performed using the WIDTH9 code (Kurucz 1993) with except for mercury and phosphorus which showed only blended lines that were reproduced with SYNTHE. Abundances are expressed in the form $\log N_{\mathrm{el}} / N_{\text {tot }}$.

Within the experimental errors, the two components show almost solar abundances so that our choice to adopt the solar opacity in the model calculation is justified. A few lines of magnesium and silicon have been detected in our spectra. According to the corrected equivalent widths, their respective abundances are slightly lower than the standard values. Still, there are elements which have significantly different abundances in the atmospheres of HD 191110 A \& B.

Phosphorus - Only one line has been observed in our spectral range, namely PII $\lambda 4499.230$. This line has been reproduced with SYNTHE considering an abundance of -5.15 and -4.70 dex for primary and secondary respectively. Both components are then overabundant with respect to the Sun.

Manganese - Abundances are very different in the atmosphere of the two components. Lines of MnI and MnII have been observed only in component A. Averaging abundances computed from these ions we get -4.80 , that is $\approx 1.9$ dex larger than the Sun's value. As reported in a series of papers by Adelman and co-workers (see for example the summarizing Table 7 of Adelman et al. 1998), this value is typical for $\mathrm{HgMn}$ stars hotter by at least $1000 \mathrm{~K}$ than HD $191110 \mathrm{~A}$.
Chromium, strontium and zirconium - The abundances of $\mathrm{Cr}$ are slightly different between the two components. The primary shows solar abundance, while the secondary is $\approx 0.3 \mathrm{dex}$ underabundant. As to strontium, the line at $\lambda 4162 \AA$ has been observed in the spectra of the secondary only. The inferred abundance is about 2 dex larger than the solar value. Lines of ZrII have been identified only in the primary's spectrum. The resulting abundance is more than 1 dex larger than the solar value. Abundances for these species are in good agreement with those measured in the atmosphere of $\chi$ Lup A (Wahlgren et al. 1994) which has effective temperature and gravity close to HD 191110.

Nickel - Only one line has been observed in the primary's spectrum. The corrected equivalent width is compatible with an overabundance of about 0.5 dex with respect to the Sun.

Yttrium and platinum - While Dworetsky (1974) reported the presence of YII and PtII lines only in the secondary's spectrum, from our analysis we found that both components show overabundances of these elements with respect to the standard values.

Neodynium - Due to the low ionization potentials of the rare-earths elements, $\mathrm{HgMn}$ stars are expected to show very weak or no singly-ionized spectral lines of those elements. On the contrary, these stars should have strong doubly-ionized lines for rare-earths.

We have identified two lines of NdIII only in the spectrum of HD $191110 \mathrm{~B}$, namely $\lambda 4921.043 \AA$ and $\lambda 4927.487 \AA$ (Fig. 3). Atomic parameters, such as $\log g f$, energies and quantum numbers for involved levels, are from Zhang et al. (2002) 
while transitions wavelengths are from Aldénius (2001). The derived abundance is -7.80 .

This abundance is in agreement with the recent results reported by Dolk et al. (2002), who studied the behavior of Neodynium in a sample of HgMn stars in the attempt to seek a correlation of $\mathrm{Nd}$ or $\mathrm{Pr}$ abundances with effective temperatures. From their sample, HR 7775 has $T_{\text {eff }}$ and $\mathrm{Nd}$ abundance comparable with those we found for HD $191110 \mathrm{~B}$.

Gold - Just one line has been detected, only in the secondary's spectrum; it is compatible with an overabundance of about 4.5 dex relative to the solar case.

As an example of the goodness of our results, we compared the observed spectrum with a synthetic spectrum computed for the adopted stellar parameters and derived abundances. Figure 3 shows this comparison in the spectral region between $4920 \AA$ and $4928 \AA$ and Fig. 4 between $4500 \AA$ and $4517 \AA$.

\subsection{Mercury}

Since the early work of Bidelman (1966) the different centroid wavelengths of the HgII $\lambda 3984 \AA$ observed in HgMn stars, were explained in terms of isotope shift due to the different isotopic composition. The profile of $\operatorname{HgII} \lambda 3984 \AA$ can be a blend of seven stable isotopes of mercury $(A=196,198,199,200,201$, $202,204)$ of which those with odd isotope numbers are further split into hyperfine components (Dworetsky et al. 1970). It is commonly accepted that the isotopic mixture can be characterized according to the $q$ parameter formalism introduced by White et al. (1976). In this formalism $q$ ranges from 0 , corresponding to the terrestrial mixture, to 4 for pure ${ }^{204} \mathrm{Hg}$. The line baricenter is shifted continuously toward longer wavelengths as $q$ increases.

To reproduce the HgII $3984 \AA$ A profile in HD 191110 A \& $\mathrm{B}$, we calculated synthetic profiles. As noted by Cowley \& Aikman (1975), this line is contaminated by several blendings, viz. FeI $\lambda 3983.956 \AA$ and $\operatorname{CrI} \lambda 3983.896 \AA$. This problem becomes worse in the case of a binary system for which each line could be contaminated by lines belonging to the other component. For example HgII line of HD 191110 B is blended with the YII $13982.594 \AA$ of component A. Thus, in our synthetic calculation we included the contributions of these nearby lines using the abundances derived from the equivalent widths measured from other unblended lines and we also included the whole spectrum of the other component.

In our procedure, we continuously changed $q$ until the corresponding $\log g f$ allowed us to achieve a good fit of the synthetic profiles to the observations. The effective oscillator strengths and wavelengths for mercury isotope lines are taken by interpolating the Smith (1997) values. As this author stated, the spectra-synthesis approach offers a clear advantage in the cases where the line is perturbed by blends. We found $q=0.2$ and $q=1.0$ for respectively the primary and secondary component of HD 191110. The result of our fit is shown in Fig. 5. The derived abundances are -5.70 for both components.

Firstly noted by White et al. (1976), the anti-correlation existing between $q$ and $T_{\text {eff }}$ was confirmed by Smith (1997).

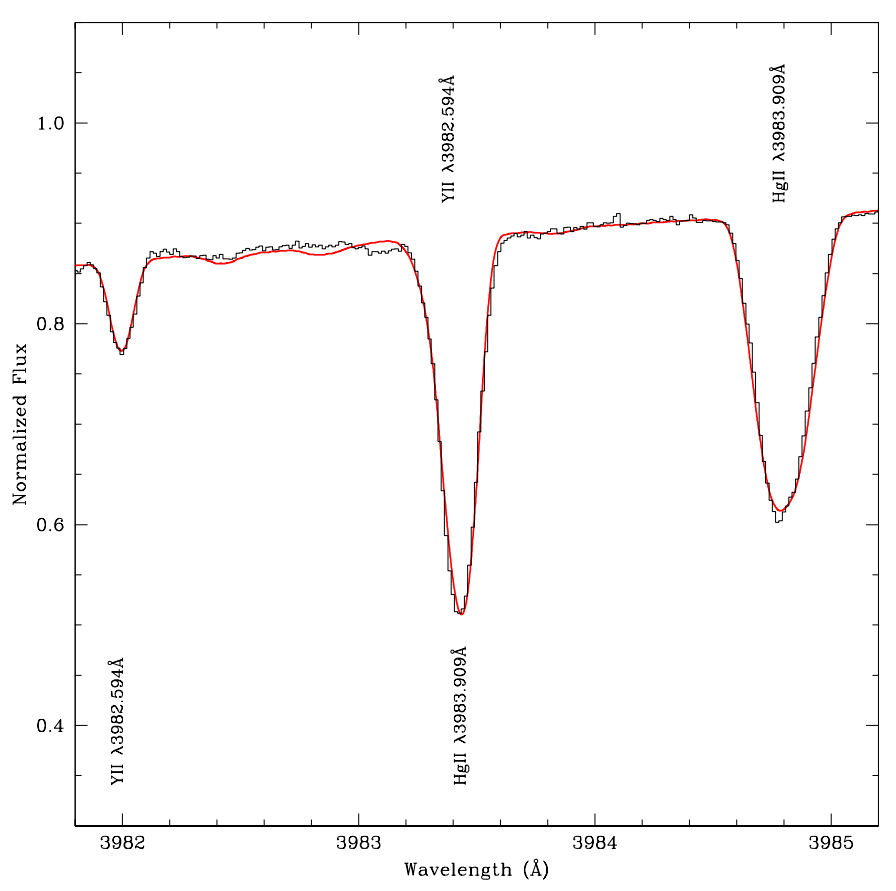

Fig. 5. Synthetic spectra (solid line) centered on the HgII $\lambda$ features compared with the observations (histogram). The total mercury abundances and isotopic-mix parameter have been properly adjusted to fit the observed profiles. The HgII line of the B component is blended with the YII line of the A component. Labels above the continuum refer to the primary and those below the spectrum to the secondary.

Referring to Fig. 2 of that paper, we see that HD 191110 B $\left(q=1.0, T_{\mathrm{eff}}=10900 \mathrm{~K}\right)$ takes up the expected position in the graph; HD 191110 A however $\left(q=0.2, T_{\text {eff }}=10800 \mathrm{~K}\right)$ showed a discrepancy of about $\delta q \approx-1$ with respect to the estimated value. However, it is worthwhile to note that in the region of lower temperature, deviations of similar quantities from the observed fit have been observed for other stars (i.e. $28 \mathrm{Her}$, $\phi$ Phe and $\chi$ Lup).

\section{Conclusion}

Using spectroscopic observations carried out at the Catania Astrophysical Observatory (low resolution) and archive data downloaded from the Canada France Hawaii Telescope archive (high resolution), we studied the double-lined spectroscopic binary with HgMn and Hg component HD 191110. The low-resolution spectra have been also used to determine the stellar parameters for both components reported in the last column of Table 3 by matching the $\mathrm{H}_{\beta}$ line profiles observed at different orbital phases.

$\mathrm{HgMn}$ in SB2 systems with temperatures and gravities similar to HD 191110 are: 112 Her (Ryabchikova et al. 1996), HR 4072 (Adelman 1994), × Lup (Wahlgren et al. 1994), 46 Dra (Adelman et al. 1998), AR Aur (Khokhlova et al. 1995, Adelman et al. 1998) and HD 32964 (Yushchenko et al. 1999). In Table 4 we compared abundances we found for HD 191110 A \& B with those inferred for these systems. From this comparison we concluded that HD 191110 presents no anomalous values. For example, platinum has very different abundances in HD 191110 A \& B: the secondary is richer by more 
Table 4. Abundances derived for HD 191110 A \& B compared with those inferred by several authors (see text for references) in similar systems. As those abundances are expressed in terms of $\log N(\mathrm{el}) / N(\mathrm{H})$, to directly compare them with our results we have to take into account the correction due to the helium abundance, adding 0.017 dex to the abundances of the primary and 0.004 dex to those of the secondary. Abundances marked with a colon should be considered as upper limits. For each star we reported the inferred $T_{\text {eff }}$ and $\log g$. In the last column we report the solar abundances (Grevesse et al. 1996).

\begin{tabular}{|c|c|c|c|c|c|c|c|c|c|c|c|c|c|c|c|}
\hline \multirow[t]{4}{*}{ El. } & \multicolumn{2}{|c|}{ HD 191110} & \multicolumn{2}{|c|}{$112 \mathrm{Her}$} & \multicolumn{2}{|c|}{46 Dra } & \multicolumn{2}{|c|}{ HR 4072} & \multicolumn{2}{|c|}{$\chi$ Lup } & \multicolumn{2}{|c|}{ AR Aur } & \multicolumn{2}{|c|}{ HD 32964} & \multirow[t]{4}{*}{ Sun } \\
\hline & A & B & A & B & A & B & A & B & A & B & A & B & A & B & \\
\hline & 11000 & 10700 & 13100 & 8500 & 11700 & 11100 & 10900 & 8900 & 10650 & 9200 & 10950 & 10350 & 11100 & 10900 & \\
\hline & 3.60 & 3.90 & 4.21 & 4.20 & 4.11 & 4.11 & 4.07 & 4.20 & 3.90 & 4.00 & 4.33 & 4.28 & 4.25 & 4.25 & \\
\hline HeI & -1.39 : & -2.00 & -2.43 & & -2.00 & -1.78 & -1.46 & & -1.68 & & & & -1.02 & -1.02 & -1.01 \\
\hline MgI & -4.64 & -4.58 & -5.56 & -5.25 & -5.19 & -4.63 & -4.65 & -5.12 & -4.48 & -4.66 & & & -4.51 & -4.85 & -4.42 \\
\hline SiII & -4.35 & -4.31 & -5.05 & & -4.82 & -4.55 & -4.53 & -5.18 & -4.33 & -4.56 & -4.24 & -4.21 & -5.03 & -5.13 & -4.45 \\
\hline PII & -5.15 & -4.70 & -4.71 & & -5.11 & -5.55 & -5.54 & & -5.68 & & & & & & -6.55 \\
\hline SII & -4.78 & -4.33 & -5.84 & & -6.00 & $:-4.95$ & -4.64 & & -4.36 & & -4.70 & & -4.66 & -4.74 & -4.67 \\
\hline TiII & -7.21 & -6.92 & -6.30 & -6.79 & -6.73 & -6.48 & -6.16 & -7.06 & -6.54 & -6.93 & -6.26 & -6.67 & -6.79 & -6.09 & -6.98 \\
\hline CrII & -6.28 & -6.05 & -6.50 & -5.94 & -6.38 & -5.95 & -5.62 & -6.16 & -6.18 & -6.09 & -5.82 & -5.79 & -6.16 & -5.66 & -6.33 \\
\hline MnI & -4.95 & & -4.74 & -5.97 & -5.33 & -5.68 & -5.51 & -6.51 & -6.78 & -6.70 & & & & & -6.61 \\
\hline MnII & -4.67 & & -4.91 & -6.00 & -5.14 & -6.11 & -5.38 & -5.97 & -6.38 & -6.63 & -5.08 & -5.67 & -6.00 & -5.61 & -6.61 \\
\hline FeI & -4.95 & -4.48 & -3.55 & -4.44 & -4.08 & -4.09 & -4.00 & -4.51 & -4.30 & -4.73 & -3.86 & -4.03 & -4.21 & -4.45 & -4.50 \\
\hline FeII & -4.43 & -4.23 & -3.60 & -4.45 & -4.15 & -4.20 & -4.08 & -4.54 & -4.34 & -4.51 & -3.82 & -4.05 & -4.28 & -4.34 & -4.50 \\
\hline NiII & -5.29 & & & -5.18 & -6.29 & & -6.62 & -5.45 & -6.08 & -5.42 & -6.93 & -5.16 & & -5.38 & -5.75 \\
\hline SrII & & -6.92 & -8.57 & -8.70 & -8.00 & -6.87 & -6.49 & -8.50 & -7.03 & -8.71 & -6.79 & -8.13 & & & -9.03 \\
\hline YII & -7.33 & -7.98 & -8.04 & -8.71 & -7.91 & -7.78 & -6.56 & -8.64 & -8.08 & -9.28 & -7.14 & -8.67 & & -6.79 & -9.76 \\
\hline ZrII & -8.32 & & -7.74 & -8.74 & -7.58 & -8.17 & -8.04 & -8.99 & -8.88 & -9.05 & -7.59 & -8.62 & & -7.81 & -9.40 \\
\hline NdIII & & -7.80 & & & & & & & & & & & & & -10.50 \\
\hline PtII & -5.96 & -4.88 & & & -6.93 & -5.72 & -5.77 & & -6.23 & & -4.97 & -5.74 & & -5.25 & -10.20 \\
\hline AuII & & -6.53 & & & -7.58 & -7.23 & -7.34 & & -7.47 & & & & & -5.34 & -10.99 \\
\hline HgII & -5.70 & -5.70 & -6.00 & & -5.80 & -5.50 & -5.28 & & -5.60 & & -6.25 & & & -5.60 & -10.83 \\
\hline
\end{tabular}

than 1.0 dex relative to the primary. The same behavior has been observed in 46 Dra, while in AR Aur the primary is more abundant than the companion. The HD 191110 primary is, instead, richer in yttrium than the secondary by about 0.6 dex. With the exception of 46 Dra, this behavior is also observed in the other systems.

Various correlations among element abundances and effective temperature are given in the literature (see for example Adelman et al. 2001). In particular, according to the correlation between $\mathrm{Mn}$ abundance and $T_{\text {eff }}$, the hotter the star more abundant the Mn. The abundance of manganese we found for HD $191110 \mathrm{~A}$ is typical for stars that are about $1000 \mathrm{~K}$ hotter (for example for $v \operatorname{Her}\left(T_{\mathrm{eff}}=11950 \mathrm{~K}, \log g=3.70\right.$ ) Adelman et al. (2001) gave the value -4.75 for the MnII abundance), which agrees approximately with the effective temperature we adopted ignoring the helium peculiarity (first column of Table 3). According to our analysis, all the effective temperatures should be lower by an amount depending on the helium abundance and then all the correlations studied should be revisited.

Acknowledgements. This research has made use of the SIMBAD database, operated at CDS, Strasburg, France.

Thanks are due to the referee, Dr. F. Carrier, for calling our attention to the test of significance for elliptical orbits developed by Lucy \& Sweeney. 


\section{Appendix A: Measured equivalent widths}

Table A.1. Spectral lines identified for HD 191110 A and B. For each chemical element we reported the laboratory wavelength, the observed and corrected equivalent widths and the derived abundance compared with that observed in the Sun. The equivalent widths are expressed in $\mathrm{m} \AA$ and the abundances in terms of $\log N_{\mathrm{el}} / N_{\mathrm{tot}}$. Values expressed in parentheses are uncertain. Solar abundances are from Grevesse et al. (1996).

\begin{tabular}{|c|c|c|c|c|c|}
\hline \multirow[t]{2}{*}{$\lambda(\AA)$} & \multicolumn{2}{|c|}{ HD $191110 \mathrm{~A}$} & \multicolumn{2}{|c|}{ HD $191110 \mathrm{~B}$} & \multirow[t]{2}{*}{ Sun } \\
\hline & $W^{\text {obs }}$ & $W^{\text {corr }}$ & $W^{\text {obs }}$ & $W^{\text {corr }}$ & \\
\hline MgI & \multicolumn{2}{|c|}{-4.64} & \multicolumn{2}{|c|}{-4.58} & -4.42 \\
\hline 5183.604 & 34.5 & 63.3 & 28.0 & 61.5 & \\
\hline SiII & \multicolumn{2}{|c|}{$-4.35 \pm 0.14$} & \multicolumn{2}{|c|}{$-4.31 \pm 0.06$} & -4.45 \\
\hline 5041.024 & 57.3 & 105.0 & 41.2 & 90.5 & \\
\hline 5055.984 & 84.9 & 155.6 & 55.8 & 122.7 & \\
\hline SII & \multicolumn{2}{|c|}{$-4.78 \pm 0.30$} & \multicolumn{2}{|c|}{-4.33} & -4.67 \\
\hline 4153.068 & 5.9 & 10.8 & 4.5 & 9.8 & \\
\hline 4924.110 & 3.4 & 6.2 & - & - & \\
\hline TiII & \multicolumn{2}{|c|}{$-7.21 \pm 0.16$} & \multicolumn{2}{|c|}{$-6.92 \pm 0.07$} & -6.98 \\
\hline 4053.834 & 6.0 & 11.0 & 7.0 & 15.4 & \\
\hline 4163.648 & 13.3 & 24.3 & - & - & \\
\hline 4501.273 & 15.9 & 29.1 & 21.1 & 46.5 & \\
\hline CrII & \multicolumn{2}{|c|}{$-6.28 \pm 0.08$} & \multicolumn{2}{|c|}{$-6.05 \pm 0.05$} & -6.33 \\
\hline 3979.505 & 9.2 & 16.9 & 10.1 & 22.3 & \\
\hline 4037.972 & 7.5 & 13.7 & - & - & \\
\hline 4049.097 & 4.2 & 7.6 & 4.3 & 9.6 & \\
\hline 4051.930 & 7.0 & 12.8 & - & - & \\
\hline 4054.076 & 5.6 & 10.2 & 5.5 & 12.1 & \\
\hline 4179.421 & 7.2 & 13.2 & - & - & \\
\hline 4912.462 & 3.0 & 5.5 & - & - & \\
\hline MnI & \multicolumn{2}{|c|}{$-4.95 \pm 0.24$} & & & -6.61 \\
\hline 4041.355 & 14.9 & 27.2 & - & - & \\
\hline 4055.544 & 6.3 & 11.6 & - & - & \\
\hline 4502.213 & 2.3 & 4.3 & - & - & \\
\hline MnII & \multicolumn{2}{|c|}{$-4.67 \pm 0.22$} & & & -6.61 \\
\hline 3778.318 & 33.5 & 61.3 & - & - & \\
\hline 3994.119 & 5.3 & 9.7 & - & - & \\
\hline 4171.512 & 15.8 & 28.9 & - & - & \\
\hline 4174.318 & 16.5 & 30.3 & - & - & \\
\hline 4497.941 & 7.0 & 12.9 & - & - & \\
\hline 4500.543 & 10.6 & 19.5 & - & - & \\
\hline 4503.201 & 10.5 & 19.3 & - & - & \\
\hline 4518.956 & 21.0 & 38.5 & - & - & \\
\hline 4525.326 & 19.2 & 35.3 & - & - & \\
\hline 4905.618 & 6.7 & 12.2 & - & - & \\
\hline 5102.517 & 31.5 & 57.7 & - & - & \\
\hline FeI & \multicolumn{2}{|c|}{$-4.95 \pm 0.12$} & \multicolumn{2}{|c|}{$-4.48 \pm 0.11$} & -4.50 \\
\hline 4045.813 & 11.5 & 21.0 & 19.2 & 42.3 & \\
\hline 4063.594 & 11.7 & 21.4 & 18.4 & 40.5 & \\
\hline 4920.502 & - & - & 11.1 & 24.5 & \\
\hline
\end{tabular}

Table A.1. Continued.

\begin{tabular}{|c|c|c|c|c|c|}
\hline \multirow[t]{2}{*}{$\overline{\lambda \lambda(\AA)}$} & \multicolumn{2}{|c|}{ HD 191110A } & \multicolumn{2}{|c|}{ HD 191110B } & \multirow[t]{2}{*}{ Sun } \\
\hline & $W^{\text {obs }}$ & $W^{\text {corr }}$ & $W^{\mathrm{obs}}$ & $W^{\text {corr }}$ & \\
\hline FeII & \multicolumn{2}{|c|}{$-4.43 \pm 0.20$} & \multicolumn{2}{|c|}{$-4.23 \pm 0.13$} & -4.50 \\
\hline 3783.347 & 14.0 & 25.7 & 13.6 & 30.0 & \\
\hline 4041.641 & 1.4 & 2.5 & - & - & \\
\hline 4044.012 & 9.7 & 17.8 & 6.5 & 14.4 & \\
\hline 4048.832 & - & - & 10.2 & 22.5 & \\
\hline 4057.461 & 7.7 & 14.1 & 9.9 & 21.7 & \\
\hline 4173.461 & 29.2 & 53.6 & 34.9 & 76.7 & \\
\hline 4178.862 & 29.3 & 53.7 & 27.5 & 60.4 & \\
\hline 4499.688 & 2.8 & 5.1 & 3.1 & 6.8 & \\
\hline 4507.102 & - & - & 4.5 & 9.9 & \\
\hline 4508.288 & 30.2 & 55.5 & 29.2 & 64.3 & \\
\hline 4515.339 & 26.4 & 48.3 & - & - & \\
\hline 4520.224 & 24.3 & 44.6 & 31.5 & 69.3 & \\
\hline 4522.634 & 33.3 & 61.0 & 29.7 & 65.3 & \\
\hline 4908.151 & 6.3 & 11.6 & 5.8 & 12.7 & \\
\hline 4913.292 & 9.6 & 17.5 & 10.0 & 22.0 & \\
\hline 4923.927 & 55.9 & 102.5 & 51.8 & 113.9 & \\
\hline 5018.440 & 69.6 & 127.6 & 53.3 & 117.2 & \\
\hline 5169.033 & 70.4 & 129.1 & 53.2 & 117.1 & \\
\hline 5197.577 & - & - & 35.5 & 78.0 & \\
\hline 5234.625 & 42.2 & 77.4 & - & 一 & \\
\hline 5276.002 & 48.4 & 88.7 & 34.8 & 76.5 & \\
\hline 5362.869 & 34.4 & 63.0 & 33.0 & 72.6 & \\
\hline NiII & \multicolumn{2}{|c|}{-5.29} & & & -5.75 \\
\hline 4509.272 & 2.5 & 4.6 & - & - & \\
\hline SrII & & & \multicolumn{2}{|c|}{-6.92} & -9.03 \\
\hline 4161.792 & - & - & 13.1 & 28.8 & \\
\hline$\overline{Y I I}$ & \multicolumn{2}{|c|}{$-7.33 \pm 0.30$} & \multicolumn{2}{|c|}{$-7.98 \pm 0.19$} & -9.76 \\
\hline 3774.331 & 29.9 & 54.8 & 17.7 & 38.9 & \\
\hline 3776.559 & 18.0 & 33.0 & 7.3 & 16.1 & \\
\hline 3788.694 & 33.6 & 61.5 & 16.9 & 37.1 & \\
\hline 3982.594 & - & - & 14.6 & 32.1 & \\
\hline 4883.684 & 43.0 & 78.9 & 17.5 & 38.5 & \\
\hline 4900.120 & 36.2 & 66.4 & 18.2 & 40.0 & \\
\hline 5087.416 & 40.0 & 73.3 & 22.6 & 49.8 & \\
\hline 5205.724 & 30.5 & 56.0 & 22.9 & 50.4 & \\
\hline ZrII & \multicolumn{2}{|c|}{$-8.32 \pm 0.14$} & & & -9.40 \\
\hline 3991.152 & 6.5 & 12.0 & - & - & \\
\hline 4149.217 & 11.1 & 20.3 & - & - & \\
\hline 4161.213 & 4.8 & 8.8 & - & - & \\
\hline NdIII & & & \multicolumn{2}{|c|}{-7.80} & -10.50 \\
\hline 4921.043 & 一 & - & (5.0) & (9.15) & \\
\hline 4927.487 & - & - & 15.0 & 27.45 & \\
\hline PtII & \multicolumn{2}{|c|}{$-5.96 \pm 0.20$} & \multicolumn{2}{|c|}{$-4.88 \pm 0.35$} & -10.20 \\
\hline 4046.450 & 23.0 & 42.1 & 33.2 & 73.0 & \\
\hline 4061.660 & 14.3 & 26.2 & 22.8 & 50.1 & \\
\hline 4148.282 & 10.3 & 18.9 & - & - & \\
\hline 4514.170 & - & - & 30.3 & 66.6 & \\
\hline AuII & & & \multicolumn{2}{|c|}{-6.53} & $(-10.99)$ \\
\hline 4052.790 & - & - & 7.6 & 16.7 & \\
\hline HgII & \multicolumn{2}{|c|}{-5.70} & \multicolumn{2}{|c|}{-5.70} & $(-10.83)$ \\
\hline 3983.890 & 88.3 & 161.9 & 80.4 & 176.9 & \\
\hline
\end{tabular}




\section{References}

Adams, W. S., \& Joy, A. H. 1917, PASP, 29, 259

Adelman, S. J., Gulliver, A. F., \& Rayle, K. E. 2001, A\&A, 367, 597

Adelman, S. J. 1994, MNRAS, 266, 97

Adelman, S. J., Ryabchikova, T. A., \& Davydova E. S. 1998, MNRAS, 297, 1

Aldénius, M. 2001, Master Thesis, Department of Physics, Univ. of Lund

Aikman, G. C. L. 1976, PDAO, 14, 379

Bidelman, W. P. 1966, in Abundance Determinations in Stellar Spectra, ed. H. Hubenet (London: Academic Press), Proc. IAU Symp., 26, 229

Conti, P. S. 1970, A\&A, 7, 213

Cowley, C. R., \& Aikman, G. C. L. 1975, PASP, 87, 513

Cowley, A., Cowley, C., Jaschek, M., \& Jaschek, C. 1969, AJ, 74, 375

Dolk, L., Wahlgren, G. M., Lundberg, H., et al. 2002, A\&A, 385, 111

Dworetsky, M. M. 1974, PASP, 86, 183

Dworetsky, M. M., Ross, J. E., \& Aller, L. H. 1970, Bull. Am. Astr. Soc., 2, 311

Giménez, A., \& Zamorano, J. 1985, Ap\&SS, 114, 259

Grevesse, N., Noels, A., \& Sauval, A. J. 1996, in Cosmic Abundances, ed. S. S. Holt, \& G. Sonnerbon, ASP Conf. Ser., 99, 117

Khokhlova, V. L., Zverko, Yu., Zhizhnovskii, I., \& Griffin, R. E. M. 1995, Astron. Lett., 21, 818
Kurucz, R. L. 1993, A new opacity-sampling model atmosphere program for arbitrary abundances. In: Peculiar versus normal phenomena in A-type and related stars, IAU Colloq. 138, ed. M.M. Dworetsky, F. Castelli, \& R. Faraggiana, ASP Conf. Ser., 44, 87

Kurucz, R. L., \& Avrett, E. H. 1981, SAO Special Rep. 391

Leone, F., \& Manfrè, M. 1996, A\&A, 315, 526

Leone, F., Lanzafame, A. C., \& Pasquini, L. 1995, A\&A, 293, 457

Lucy, L. B., \& Sweeney, M. A. 1971, AJ, 76, 544

Piskunov, N. E., Kupka, F., Ryabchikova, T. A., Weiss, W. W., \& Jeffery, C. S. 1995, A\&AS, 112, 525

Ryabchikova, T. A., Zakharova, L. A., \& Adelman, S. J. 1996, MNRAS, 283, 1115

Smith, K. C. 1997, A\&A, 319, 928

Stellingwerf, R. F. 1978, ApJ, 224, 953

Wahlgren, G. M., Adelman, S. J., \& Robinson, R. D. 1994, ApJ, 434, 349

White, R. E., Vaughan, A. H., Preston, G. W., \& Swings, J. P. 1976, ApJ, 204, 131

Yushchenko, A. V., Gopka, V. F., Khokhlova, V. L., Musaev, F. A., \& Bikmaev, I. F. 1999, AstL, 25, 435

Udry, S., Mayor, M., \& Queloz, D. 1999, Precise Stellar Radial Velocities, IAU Colloq. 170, ed. J. B. Hearnshaw, \& C. D. Scarfe, ASP Conf. Ser., 185, 367

Zhang, Z. G., Svanberg, S., Palmeri, P., Quinet, P., \& Biémont, E. 2002, A\&A, 385, 724 